\title{
The biased balance: observation, formalism and interpretation of a dissymmetric measuring device*
}

\author{
Marc Le Menestrel ${ }^{\dagger}$ \\ January 2007
}

\begin{abstract}
This paper studies a balance whose unobservable fulcrum is not necessarily located at the middle of its two pans. It presents three different models, showing how this lack of symmetry modifies the observation, the formalism and the interpretation of such a biased measuring device. It argues that the biased balance can be an interesting source of inspiration for broadening the representational theory of measurement.
\end{abstract}

Keywords: representational theory of measurement, biased measurement, bias, rationality, irrationality, observer, dependence, indeterminacy, invariance

JEL: A0, B4, C0, D0.

*I am grateful to Sybille van den Hove for her sustained support in the development and clarification of the ideas expressed in this paper. I am also deeply indebted to Bertrand Lemaire without whom I would not have been able to develop my intuitions about the biased balance (in particular, the formulation and proof of Theorem 3 of this paper have benefited from his corrections). I also would like to thank Robin Hogarth, Duncan Luce, Thierry Marchant, Riccardo Pedersini, Julian Rode and Patrick Suppes for their very useful comments on earlier drafts of this paper.

†Universitat Pompeu Fabra, Departament d'Economia i Empresa, Ramon Trias Fargas 2527, 08005 Barcelona, España. e-mail: marc.lemenestrel@upf.edu. 
Then, at last, as they were nearing the fountains for the fourth time, the father of all balanced his golden scales and placed a doom in each of them, one for Achilles and the other for Hector. As he held the scales by the middle, the doom of Hector fell down deep into the house of Hades- and then Phoebus Apollo left him.

Homer, Iliad XXII

Give me a place to stand on, and I can move the earth. Archimedes

\section{Introduction}

The balance is a universal symbol for measurement and judgment. Osiris uses a balance to measure the soul of the dead in ancient Egypt. In the Greek epic tradition, deities like Apollo use a balance to decide of the fate of heroes. As a measuring device, it is discussed by Plato, Aristotle, Euclid and Archimedes (Heath 1897, Moody and Clagett 1960). It appears in the Bible as a symbol for rigor and exactness and in the Koran as a symbol of supreme wisdom. It symbolizes the invariable middle in ancient China, is part of the Sanskrit mythology and of the Indian and Tibetan spiritual traditions (Chevalier and Gheerbrant 1969). In the middle ages, the balance was essential to evaluate the price of goods and to allow for the development of trade (Kish 1965). Nowadays, it is a symbol of justice all over the modern world. It is ubiquitous in the philosophy of science (Campbell 1957, Ellis 1966, Carnap 1966) and is a seminal example for the foundations of measurement (e.g. Krantz et al. 1971, Roberts 1979, Narens 1985). Undeniably, the equal-arm balance is a powerful model for the theory of measurement and for the intuition of unbiased judgment.

To make more precise this importance, consider an equal-arm balance and position an object, denoted $x$, on one of its pan and an object $y$ on the other pan. Suppose that you observe that " $x$ is lower than $y$ ". This observation is formally described with a binary relation $\succ_{0}$ as " $x \succ_{0} y$ ". Add another object $z$ to $x$. You observe that " $x$ with $z$ are lower than $y$ ". Since this happens for any object $z$, the empirical regularity of such an observation leads to assume the following property:

$$
\text { for all } x, y, z \text { we have } x \succ_{0} y \Rightarrow(x \circ z) \succ_{0} y \text {, }
$$


where "o" naturally means the operation of jointly positioning two objects on the same pan of the balance. Further axioms then reflect the laws or regularities that can be observed, including in particular the following independence property:

$$
\text { for all } x, y, z \text { we have } x \succ_{0} y \Leftrightarrow(x \circ z) \succ_{0}(y \circ z) \text {. }
$$

With sufficient axioms, your task of measurement is then to prove the existence of a function, say $\varphi$, which assigns a number to each object such that an object is lower than another on the balance if and only if it is assigned a greater number. Formally, you prove that there exists a real-valued function $\varphi$ such that

$$
\begin{gathered}
x \succ_{0} y \Longleftrightarrow \varphi(x)>\varphi(y), \\
\varphi(x \circ y)=\varphi(x)+\varphi(y) .
\end{gathered}
$$

Such a representation theorem is likely to build on Hölder's theorem (see Michell and Ernst 1996 for an English translation) and the theory of extensive measurement (see Krantz et al. 1971, Chapter 3). Naturally, the number $\varphi(x)$ means "the mass of $x$ ". The function $\varphi$ is unique up to multiplication by a positive constant and is called a ratio-scale (see Stevens 1946). Of course, many predictions can be made based on this measurement. For instance, if the sum of the mass of $y$ and the mass of $z$ is greater than the mass of $x$, you predict with certainty that you will observe that " $y$ with $z$ is lower than $x$ ". With this simple model of the equal-arm balance, the observed relation between objects does not depend on the measuring device and does not depend on the observer. Also, the observed empirical relation is formalized with formal (non-numerical) statements which univocally correspond with observation. Finally, a numerical representation is provided which measures objects and the function alone suffices to this measurement. As we will see in this paper, things are quite different for the biased balance.

A biased balance is a two-arm balance whose fulcrum is not necessarily located at the middle of the two pans. Of course, the principle of the balance with unequal arms has long been understood, at least since Archimedes' proof of the principle of the lever (Propositions 6 and 7 of Book I of On the equilibrium of the planes, see Heath 1897 p. 192). Also, the so-called Roman or Steelyard balance, where objects positioned on a tray at one end of the beam are balanced by moving a counterweight along the opposite side of the beam, has been employed to weigh large bodies from the earliest time. Not only the principle of the lever had to be invoked, but also the account of the weight of the tray (or hook) used to hold the object to be weighed, which induces some complications (see for instance the Liber de Canonio in Moody and Clagett 1960). As shown in Suppes (1980), these earlier mathematical approaches are very close to the contemporaneous theory of conjoint measurement (Krantz et al. 1971, Chapter 6). What they share in particular is that they start with two dimensions (here weights and distances) 
which can be manipulated independently in order to observe their conjoint effect. In particular, it is assumed possible to select the distances from the fulcrum so that they are of appropriate proportions. Also, it is assumed that distances can be divided into segments of equal length.

In this study of the biased balance, we do not assume that distance from the fulcrum is an observable primitive. We rather assume that we only observe a relation among objects and we then try to characterize the implicit role of the bias. Hence, we do not start from two dimensions playing similar roles but with one that is observable (the objects positioned on the balance) and infer the role of the second that is not directly observable (the bias of the balance). Because of the hidden role of the bias, the relation between objects presents less regularity. Therefore, the axioms which formalize observation are more general than in the traditional theory of measurement. This is why the biased balance is interesting as a model to broaden the formal theory of measurement. This approach is also a way to study the specificity of the bias as a property of the measuring device rather than a property of the objects themselves. As such, it sheds light on the extent to which we can measure the bias of a measuring device from the mere observation of the behavior of the objects that such a device measures.

I present below three models of the biased balance which differ by their assumptions about what is observed and how it is observed. They present distinct empirical regularities which correspond to different set of axioms. I thus present different representation theorems which prove that, even with these irregularities, a proper numerical measure of the mass of objects exists. These theorems reveal a numerical factor which somehow "measures" these irregularities and which intuitively corresponds to the bias of the balance. As the interpretation of such number is not necessarily obvious, I make precise what it means and what it measures. The biased balance hence leads to a more detailed analysis of the correspondence between empirical observation and its formalization as a relational structure. This step is usually taken for granted in the theory of representational measurement, due to an implicit assumption of the symmetry of the measuring device. More generally, the biased balance sheds light on three fundamental questions:

1. How does empirical observation relate to its formal description?

2. What formal properties must be assumed in order to construct a numerical representation of this formal description?

3. What is the meaning of such a numerical representation with respect to the experimental setting which gives rise to empirical observation?

These questions are essential to a clear and precise understanding of the use of numbers and of mathematical models in sciences. A most complete and systematic attempt to address them is found in the representational theory of measurement. Because the biased balance shows how this theory may be broadened 
to apply to phenomena which do not present the typical empirical regularities assumed by the symmetry of the measuring device, it contributes to address one of its most interesting critique (e.g. Schwager 1991, Mari 2000, 2005, Boumans 2005).

The rest of the paper is structured as follows. In section 2, I present the basic terms and formal properties that I use to study a biased balance. I also introduce the 3 models. In section 3, I present a first model which shows some dependence on the observer. This dependence arises because the model relies on the distinction between observer's left and right. Since it is the most general model from the mathematical point of view, I present it first, so that other models can refer to its representation theorem. In section 4, I present a second model which reflects some indeterminacy in observable behavior. This indeterminacy is mainly due the assumption that the interaction between the objects and the biased balance is not directly observable. In section 5 , I present a third model where I define some "extended objects" in order to explicitly take account of the interaction with the balance. This model is a form of conjoint measurement. A short section 6 concludes.

\section{Preliminaries}

In an experiment, the behavior of a biased balance is determined by three types of considerations. First, there are the objects which are positioned on its pans. Second, there is the measuring device, the balance itself, which has a specific bias, i.e. whose fulcrum is located at a given place between the two pans. Third, there is the allocation of objects to the pans. Indeed, given two objects and given one biased balance, the behavior of the measuring device is not determined: it depends on the respective positioning of the objects on the pans. This is the interaction between the object and the balance.

We refer to observation as the visual perception of the behavior of the balance by an observer when objects are positioned on the pans. We designate observations by pictures or by observational statements. Figure 1 shows three different ways to observe a particular behavior of a biased balance, with object $x$ placed on one pan and object $y$ placed on the other pan. Each picture can be described by the following observational statements:

$\left(a_{1}\right)$ " $x$ on the left is lower than $y$ on the right";

$\left(a_{2}\right)$ " $x$ is lower than $y$ ";

$\left(a_{3}\right)$ " $x$ on pan \#1 is lower than $y$ on pan \#2".

Statement $\left(a_{1}\right)$ differs from statement $\left(a_{2}\right)$ because it specifies on which pan each object is positioned. However, it does so in a manner which depends on the observer (left and right are relative to the observer). The third statement $\left(a_{3}\right)$ 
$\left(a_{1}\right)$

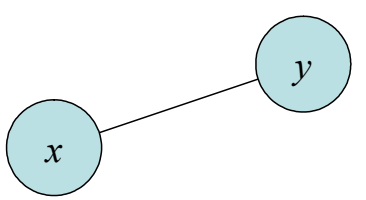

" $x$ on the left is lower than $y$ on the right" $\left(a_{2}\right)$

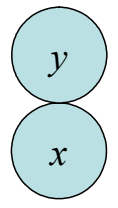

" $x$ is lower

than $y$ " $\left(a_{3}\right)$

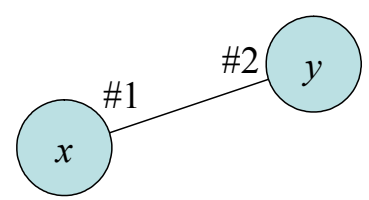

" $x$ on pan \#1 is lower than $y$ on pan \#2"

Figure 1: Three ways to describe the observation of the behavior of a biased balance

specifies on which pan each object is placed, but designates the pans in a manner independent of the observer. We see below that these three observations lead to three different models.

Let us now consider the formalization of observations. We consider a set of objects $A$ and, as we have already done, we denote objects by $x, y, z \ldots \in A$. We designate by $x \circ y$ the object consisting of two objects $x$ and $y$ and we assume that the operation $\circ$ is closed (for all $x, y \in A, x \circ y \in A$ ), commutative (for all $x, y \in A, x \circ y=y \circ x$ ) and associative (for all $x, y, z \in A, x \circ(y \circ z)=(x \circ y) \circ z)$. The set $A$ endowed with such an operation is a commutative semigroup. For $m \in \mathbb{N}^{*}$, we define $m x$ by $1 x=x$ and $m x=(m-1) x \circ x$ where $\mathbb{N}^{*}$ stands for the set of positive integers. Naturally, $m x$ designs the object consisting of $m$ copies of $x$ (note this already departs from the intuitive analysis where every object is distinct). Further, we assume that $A$ is homogeneous, i.e. that given two objects $x$ and $y$, there exist two positive integers $m$ and $n$ such that $m x=n y$ (note that this hypothesis implies that there is no object of null mass). As we did in the introduction, we formalize the behavior of the balance with a binary relation noted $\succ$ when the balance is not at equilibrium and $\sim$ when the balance is at equilibrium. To characterize the observed regularities of each model and to prove our representation theorems, we use different properties for binary relations on a commutative semigroup. Consider a binary relation $R$ on a commutative semigroup $X$, we use the following definitions:

- $R$ is asymmetric if and only if, for all $x, y \in X, x R y \Rightarrow n o t(y R x)$;

- $R$ is symmetric if and only if, for all $x, y \in X, x R y \Rightarrow y R x$;

- $R$ is complete if and only if, for all $x, y \in X, x R y$ or $y R x$;

- $R$ is transitive if and only if, for all $x, y, z \in X,(x R y$ and $y R z) \Rightarrow x R z$; 
- $R$ is positive if and only if, for all $x, y, z \in X, x R y \Rightarrow(x \circ z) R y$;

- $R$ is non-trivial if and only if, for some $x, y, z, t \in X, x R y$ and not $(z R t)$;

- $R$ is scale-invariant ${ }^{1}$ if and only if, for all $x, y \in X$ and all $m \in \mathbb{N}^{*}$, $x R y \Leftrightarrow m x R m y$;

- $R$ is independent ${ }^{2}$ if and only if, for all $x, y, z \in X, x R y \Leftrightarrow(x \circ z) R(y \circ z)$;

- $R$ is super-archimedean ${ }^{3}$ if and only if, for all $x, y \in X, x R y \Rightarrow m x R n y$ for some $m<n$, with $m, n \in \mathbb{N}^{*}$.

We also use the following definition, which characterizes the pairs for which the relation $R$ is not super-archimedean ${ }^{4}$ :

- A pair $(x, y) \in X \times X$ is balanced if and only if $x R y$ and not (mxRny) for all $m<n$, with $m, n \in \mathbb{N}^{*}$.

Formalizing the biased balance aims at clarifying the conditions under which the existence and uniqueness of a function that measures objects, i.e. which assigns a numerical value (their mass) to each of them, can be proved. Such a function is denoted by $\varphi$ and takes its values in the set of positive real numbers, denoted $\mathbb{R}_{>0}$. This process of representing a relation which formalizes an observed behavior by a relation among the numbers assigned to the objects is the one of measurement. Another objective is to clarify the extent to which we can also "measure" the measuring device itself, i.e. the bias of the balance. To this purpose, our representations will reveal a factor, denoted $\alpha$ or $\beta$ and belonging to the set of positive real numbers. What such a factor means is a matter of interpretation.

By interpretation, we refer to the correspondence between something pertaining to an experimental setting with some formal symbol or statement, and reciprocally. This comprises the formalization of a particular observation, or the empirical meaning of a formal symbol or statement. This distinction between interpretation and measurement is depicted in Figure 2, with the example of statement $\left(a_{1}\right)$, anticipating our first model below.

\footnotetext{
${ }^{1}$ This scale-invariance property is also called homotheticity and is the key structural property of a biased balance.

${ }^{2}$ This standard property is also often called monotonicity.

${ }^{3}$ This is the term used by De Miguel et al. (1996) and that we adopted in Le Menestrel and Lemaire (2006b).

${ }^{4}$ This property is inspired from the property of anomalous pairs in Fuchs (1963).
} 


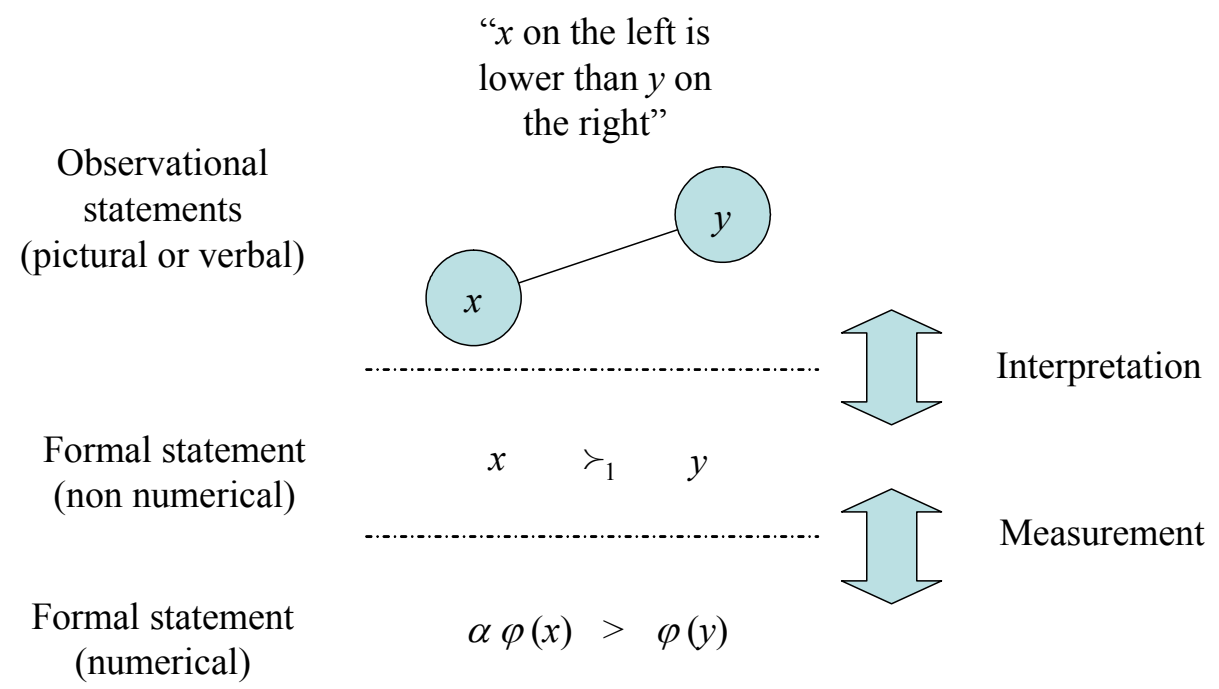

Figure 2: Interpretation and Measurement

\section{First model: A dependence on the observer}

Consider a biased balance placed in front of you, the observer. Take two objects $x$ and $y$ that are positioned on the left and right pan respectively. As pictured in Figure 3, you may observe $\left(a_{1}\right)$, i.e. that " $x$ on the left pan is lower than $y$ is on the right pan". This is formally written $x \succ_{1} y$. You may observe $\left(b_{1}\right)$, i.e. that "the balance is at equilibrium when $x$ is on the left pan and $y$ on the right pan". This is written $x \sim_{1} y$. Both $\succ_{1}$ and $\sim_{1}$ are binary relations defined on the set of objects $A$. If you observe neither of these two, then you must observe $\left(c_{1}\right)$, i.e. that " $y$ on the right pan is lower than $x$ is on the left pan". If $\succsim_{1}$ is defined as $x \succsim_{1} y \Leftrightarrow\left(x \succ_{1} y\right.$ or $\left.x \sim_{1} y\right)$, it formalizes that " $x$ on the left pan is lower when $y$ is on the right pan or the balance is at equilibrium". Therefore, you can simply formalize that $y$ on the right is lower than $x$ as $x \beth_{1} y$.

Intuitively, the relation $\succ_{1}$ is positive, reflecting that mass is a positive quantity. Also, both relations $\succ_{1}$ and $\sim_{1}$ are scale-invariant: the behavior will not change if you take $m$ copies of $x$ and of $y\left(m \in \mathbb{N}^{*}\right)$. Further, the relation $\succ_{1}$ is super-archimedean but $\sim_{1}$ is not. Indeed, if the balance is at equilibrium, it will tilt as soon as the ratio of the number of copies is modified (below, we will use this distinctive property to start from $\succsim_{1}$ and define the two relations $\succ_{1}$ and $\sim_{1}$ from this primitive). Finally, we assume that the relation $\succsim_{1}$ is non-trivial, i.e. that there exist at least two objects such that the balance tilts on the left and two objects such that the balance tilts on the right.

What is surprising is that the relation $\succ_{1}$ is not necessarily asymmetric. We would have $\left(x \succ_{1} y\right.$ and $\left.y \succ_{1} x\right)$ when, for instance, $x$ and $y$ have the same mass 
$\left(a_{1}\right)$

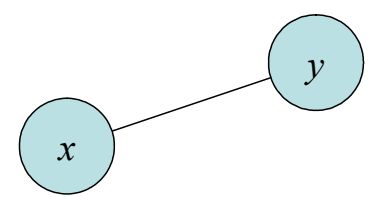

" $x$ on the left is lower than $y$ on the right"

$$
x \succ_{1} y
$$

$\left(b_{1}\right)$

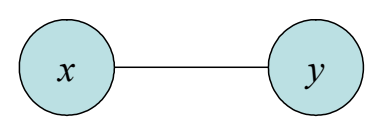

"the balance is at equilibrium when $x$ is on the left and $y$ is on the right" $\left(c_{1}\right)$

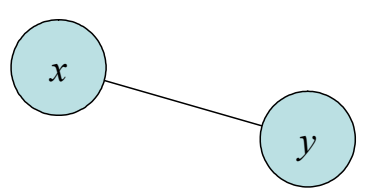

" $y$ on the right is lower than $x$ on the left"

$x \nvdash_{1} y$

Figure 3: The 3 possible outcomes of an experiment with a biased balance (model 1)

and the left arm is longer. As for the relation $\sim_{1}$, it is not necessarily symmetric: we may have $\left(x \sim_{1} y\right.$ and $\left.y \chi_{1} x\right)$. This will happen when arms have different lengths. Also, the relations $\succ_{1}$ and $\sim_{1}$ are not necessarily transitive: when the left arm is longer, we may have $\left(x \succ_{1} y, y \succ_{1} z\right.$ and $\left.x \succ_{1} z\right)$ and also $\left(x \sim_{1} y\right.$, $y \sim_{1} z$ and $\left.x \chi_{1} z\right)$. Further, the relation $\succsim_{1}$ is not necessarily complete: we may have $\left(x \beth_{1} y\right.$ and $\left.y Z_{1} x\right)$. This will happen, for instance, if $x$ and $y$ have the same mass and the right arm is longer. Finally, the relation $\succsim_{1}$ is not necessarily independent because of a possible lever effect.

In terms of the formal properties of the primitive relations $\succ_{1}$ and $\sim_{1}$, this model is thus very general. Despite this generality, we can prove the existence and uniqueness of a numerical function that measures the mass of the objects. We can also provide some sort of measurement of the bias of the balance. This is shown in the following representation theorem:

Theorem 1 Let $A$ be a commutative semigroup endowed with a nontrivial binary relation $\succsim_{1}$ that is positive and scale-invariant. Write $x \sim_{1} y$ if and only if $(x, y)$ is balanced and $x \succ_{1} y$ if and only if $\left(x \succsim_{1} y\right.$ and $\left.x \chi_{1} y\right)$. Suppose $A$ is homogeneous. Then there exist a function $\varphi: A \rightarrow \mathbb{R}_{>0}$ and a number $\alpha>0$ such that we have

$$
\begin{gathered}
x \succ_{1} y \Longleftrightarrow \alpha \varphi(x)>\varphi(y), \\
x \sim_{1} y \Longleftrightarrow \alpha \varphi(x)=\varphi(y), \\
\varphi(x \circ y)=\varphi(x)+\varphi(y) .
\end{gathered}
$$

Moreover, $\varphi$ is unique up to multiplication by a positive constant and $\alpha$ is unique. 
Proof. Suppose there is no balanced pair, then the theorem above amounts to Theorem 1 in Le Menestrel \& Lemaire (2006b). If there are balanced pairs, then $\succsim_{1}$ is not super-archimedean and we can use Theorem 2 in Le Menestrel $\&$ Lemaire (2006b). The additive property $(i i)$ is easily obtained from the homogeneity property. To see this, take $x, y \in A$. There exist $m, n$ such that $m x=n y$. Hence, we have $\varphi(x \circ y)=\frac{1}{n} \varphi(n x \circ n y)=\frac{1}{n} \varphi(n x \circ m x)=\frac{n+m}{n} \varphi(x)=$ $\varphi(x)+\frac{1}{n} \varphi(m x)=\varphi(x)+\varphi(y)$.

A simple corollary of this theorem implies that, in a homogeneous setting, any positive and scale-invariant relation is either asymmetric and transitive, or complete. It is transitive and complete (and then not asymmetric) if and only if the balance is not biased. Also, note that if there are no balanced pairs, no equilibrium can be observed. In that case, the relation $\sim_{1}$ is empty and we have $\succsim_{1}=\succ_{1}$. In this homogeneous setting, the factor $\alpha$ is then necessarily an irrational number ${ }^{5}$.

The function $\varphi$ is naturally interpreted as measuring the mass of the objects. Contrary to Mari (2000), it is not because the primitive relation is intransitive that numerical measurement is necessarily impossible. Indeed, Theorem 1 shows that measurement of the ratio of two masses is possible, in line with the definition of measurement given by Michell (2005, p. 287).

For you, the observer, if the number $\alpha$ measures the distance between the fulcrum and the left pan, then the distance between the fulcrum and the right pan is 1 . These lengths are unique up to multiplication by a positive constant: they constitute a ratio-scale.

The factor $\alpha$ cannot be directly interpreted as measuring "the bias of the balance" because the same experiment (i.e. same objects, same balance, same interaction) may lead to another formalization. Consider an observer $1^{\prime}$ placed on the other side of the balance. He would follow the same instructions to formalize his observations. However, when you observe outcome $\left(a_{1}\right)$ and formally write $x \succ_{1} y$, he would observe outcome $\left(c_{1}\right)$ and write $y \succsim_{1^{\prime}} x$. In his representation, he would obtain a factor $\alpha^{\prime}=\frac{1}{\alpha}$ that he would wrongly interpret as measuring "the bias of the balance". Because "the bias of the balance" may take two distinct values, the numerical factor $\alpha$ shall rather be interpreted as reflecting "the bias of the balance from the point of view of the observer".

Note that such a dependence can be avoided by formalizing that an object tilts the balance independently of the pan on which it is positioned. Suppose that we observe that $x$ on the left is lower than $y$ on the right and that $x$ on the right is lower than $y$ on the left. We then write $x \gg_{1} y$ (formally, we

\footnotetext{
${ }^{5}$ Reciprocally, we show in Le Menestrel and Lemaire 2006b that if $\alpha$ is irrational, then no equilibrium exists. Note also that in the absence of equilibrium, i.e. if and only if $\alpha$ is irrational, we cannot be certain with a finite number of observations that two objects have the same mass in a homogeneous setting. Hence, the assumption that we dispose of identical copies of an object $x$ becomes especially important.
} 


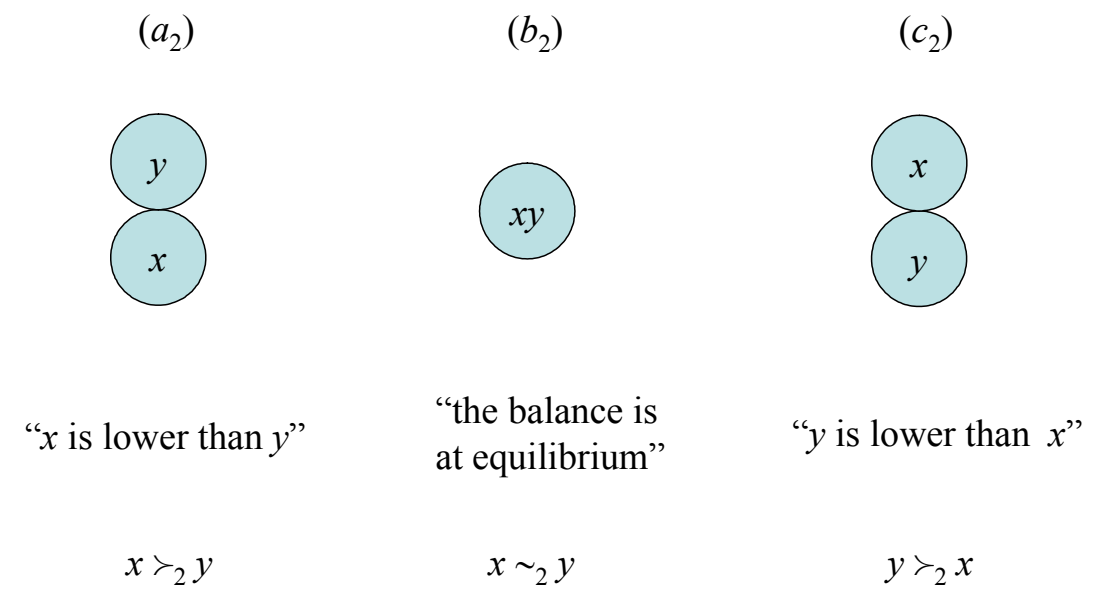

Figure 4: The 3 possible observations of an experiment with a biased balance (model 2)

have $x \gg_{1} y \Leftrightarrow\left(x \succ_{1} y\right.$ and $\left.\left.y \swarrow_{1} x\right)\right)$. The relation $\gg_{1}$ does not have the same properties than the relation $\succ_{1}$ : it is necessarily asymmetric and transitive. Hence, the factor $\alpha$ is necessarily lower or equal to 1 and the corresponding representation is $x \gg_{1} y \Longleftrightarrow \alpha \varphi(x)>\varphi(y)$ with $0<\alpha \leqslant 1$. An example of interpretation of $\gg_{1}$ distinct from the biased balance consists in formalizing the weighing of objects by hand. One tends to permute objects in the hands in order to get rid of a possible bias when assessing that an object has a greater mass than the other. The procedure leaves out objects whose masses are close, and this lack of discrimination results in a form of "intransitive indifference" with a proportional threshold of just noticeable difference referred to as Weber's law (See Le Menestrel and Lemaire 2004 who refer to this interpretation. See also the models in Le Menestrel 2006a and Lemaire and Le Menestrel 2006).

\section{Second model: An indeterminacy in observ- able behavior}

We consider next a balance placed parallel to your axis of vision. You the observer see which object is lower, if any, but do not observe on which pan each object is positioned. When object $x$ is placed on one pan of the balance and object $y$ on the other pan, you may observe $\left(a_{2}\right)$, i.e. that " $x$ is lower than $y$ ", and you would write it as $x \succ_{2} y$ (Figure 4). You may observe $\left(b_{2}\right)$, i.e. that "the balance is at equilibrium", that you would formalize as $x \sim_{2} y$. Finally, you may observe $\left(c_{2}\right)$, i.e. that " $y$ is lower than $x$ " which you would write $y \succ_{2} x$. Compared with the previous model, the distinction left and right does not apply. Because you 
cannot observe on which pan each object is placed, it is not possible to control the permutation of two objects when preparing an experiment.

Suppose that you observe that object $x$ is lower than object $y$. Of course, it does not mean that the mass of $x$ is greater than the mass of $y$. Suppose now that you make a second observation, and that this time, with the same balance and the same objects, you observe that $x$ and $y$ are at equilibrium. Then, you can infer that $x$ indeed has a greater mass than $y$, that $x$ was positioned on the longer pan in the first experiment, and that $x$ was positioned on the shorter pan in the second experiment. From two distinct observations, one being an equilibrium, we just showed how to acquire some information about the interaction between the objects and the measuring device, even though such interaction is not directly observable. Two such observations allow to make deterministic predictions. For instance, the combination of $x$ and another object is necessarily lower than $y$. Note also that if you had observed $y$ lower than $x$ in the second experiment, you could only have inferred that $x$ and $y$ have masses that do not differ "too much", without gaining information about the interaction with the measuring device nor being able to make deterministic predictions.

Formally, the relation $\succsim_{2}$ designates the union of the relations $\succ_{2}$ and $\sim_{2}$ and can again be defined as $x \succsim_{2} y \Leftrightarrow\left(x \succ_{2}\right.$ y or $\left.x \sim_{2} y\right)$. As in model 1 , the relation $\succ_{2}$ is positive and super-archimedean, and the relations $\succ_{2}$ and $\sim_{2}$ are scaleinvariant. The relation $\succ_{2}$ is not necessarily asymmetric and neither $\succ_{2}$ nor $\sim_{2}$ are necessarily transitive. On the other hand, the relation $\sim_{2}$ is symmetric, reflecting that we cannot distinguish between objects at equilibrium. Also, the relation $\succsim_{2}$ is complete (either one object tilts the balance, or the balance is at equilibrium).

Even if it does not depend on the observer, this model has less mathematical generality than model 1 . Its representation, which derives from the representation of the first model, reveals a factor greater or equal to 1 :

Theorem 2 Let $A$ be a commutative semigroup endowed with a nontrivial binary relation $\succsim_{2}$ that is positive, scale-invariant and complete. Write $x \sim_{2} y$ if and only if $(x, y)$ is balanced or $(y, x)$ is balanced, and $x \succ_{2} y$ if and only if ( $x \succsim_{2} y$ and $(x, y)$ is not balanced). Suppose $A$ is homogeneous. Then there exist a function $\varphi: A \rightarrow \mathbb{R}_{>0}$ and a number $\alpha \geqslant 1$ such that we have

$$
\begin{gathered}
x \succ_{2} y \Longleftrightarrow \alpha \varphi(x)>\varphi(y), \\
x \sim_{2} y \Leftrightarrow\left\{\begin{array}{l}
\alpha \varphi(x)=\varphi(y) \\
o r \\
\varphi(x)=\alpha \varphi(y)
\end{array},\right. \\
\varphi(x \circ y)=\varphi(x)+\varphi(y) .
\end{gathered}
$$

Moreover, $\varphi$ is unique up to multiplication by a positive constant and $\alpha$ is unique. 


\section{Proof. This is a corollary of Theorem 1.}

Note that the symmetry of the relation $\sim_{2}$ stems from its symmetrical definition which differs from the one in model 1 . This is because $x$ and $y$ may put the balance at equilibrium while the pair $(y, x)$ is not balanced. We have the peculiar property that the relations $\succ_{2}$ and $\sim_{2}$ are not disjoint, i.e. we may have $x \succ_{2} y$ and $x \sim_{2} y$. Because of this, it is not possible in this model to know with certainty that the balance is not biased ${ }^{6}$.

The function $\varphi$ has the same interpretation as in model 1. For you the observer, if the number $\alpha$ is interpreted as measuring the longer arm, then the shorter arm is of length 1 . These lengths constitute a ratio-scale. Of course, since we cannot observe the arms, it is not possible to say which one is the longer arm or the shorter.

Apart from the cases where deterministic predictions can be made, one does not know which outcome is going to be observed in this model. This indeterminacy is not due to insufficient knowledge about the objects, nor to insufficient knowledge about the measuring device, but to a lack of knowledge about the interaction between the objects and the measuring device. What appears, from the point of view of the observer, as the same "observable cause" (two given objects on a given measuring device), does not lead to the "same observable effect." This illustrates a violation of procedural invariance where two substantially equivalent settings are not empirically equivalent (see e.g. Quine 1975). In other words, observation depends on how the measuring device treats the objects. ${ }^{7}$

Note that the three statements $x \succ_{2} y, x \sim_{2} y$ and $y \succ_{2} x$ which describe the possible observations in this model are not mutually exclusive. Hence, the correspondence between observational statements and formal statements is not one-to-one. A formal statement may have two distinct meaning in terms of observational statements. For instance, if the observational statement " $x$ is lower than $y$ " necessarily leads to the formal statement $x \succ_{2} y$, the formal statement $x \succ_{2} y$ does not mean that we will necessarily observe that " $x$ is lower than $y$ ". It merely means that observing that " $x$ is lower than $y$ " is possible. It is as if there were a time asymmetry in the sense that a formal statement can correspond either to a statement of fact (a description of an observation that took place in the past) or to a statement of possibility (a prediction of an observation that may take place in the future). ${ }^{8}$

\footnotetext{
${ }^{6}$ Here, our intuition suggests that observing an equilibrium between $x$ and $y$ in a large number of experiments probably means that the balance is not biased, and that $x$ and $y$ have the same mass. But this necessitates a probabilistic approach, with an hypothesis about the random generating process assigning objects to the pans. This would be an interesting avenue for further research with links with probabilistic models.

${ }^{7}$ This should be specifically interesting for the modeling of preferences, which are often dependant on the process by which they are constructed (e.g. Slovic, 1995, Kahneman and Tversky 2000, and also Sen 1986, Le Menestrel and Wassenhove 2001).

${ }^{8}$ Somewhat similar statements have been made in the context of quantum mechanics (e.g.
} 
$\left(a_{3}\right)$

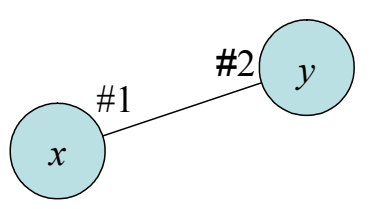

" $x$ on pan \#1 is lower than $y$ on pan \#2" $\left(b_{3}\right)$

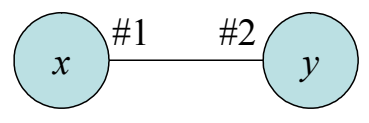

"the balance is at equilibrium when $x$ is on pan \#1 and $y$ is on pan \#2" $\left(c_{3}\right)$

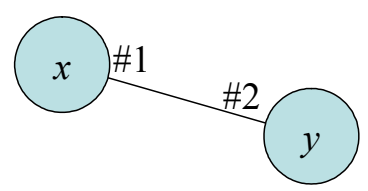
" $y$ on pan \#2 is lower
than $x$ on pan $\# 1 "$

Figure 5: The 3 possible outcomes of an experiment with a biased balance (model 3)

The relation $\succsim_{2}$ can be easily obtained from the relation $\succsim_{1}$ of the first model. Observing that $x$ is lower than $y$ means that either $x$ on the left is lower than $y$ on the right, or $x$ on the right is lower than $y$ on the left. Formally, we have $x \succsim_{2} y \Leftrightarrow\left(x \succsim_{1} y\right.$ or $\left.y \swarrow_{1} x\right)$. More precisely, the relation $\succsim_{2}$ is formally the converse of the negation of the relation $\gg_{1}$ : we have $x \succsim_{2} y \Leftrightarrow y \gg_{1} x$. Note that the relation $\gg_{1}$ itself is not directly observable in this model. However, it can be derived from observation in some particular cases. For instance, we may observe $\left(x \succ_{2} y\right.$ and $\left.y \sim_{2} x\right)$ and deduce that $x \circ z \gg_{1} y$ for all $z \in A$. In this manner, the relation $\gg_{1}$ characterizes the pairs for which a deterministic prediction can be made.

\section{Third model: Extending the definition of ob- jects to include the measuring device}

In this model, we consider an observer who can identify each pan of the balance, calling them $\# 1$ and $\# 2$. Hence, the distinction between the two pans of the balance is observable (contrary to model 2) and is independent of the observer (contrary to model 1). The principle of this model resides in extending the definition of objects in order to include their interaction with the measuring device. We consider the pair composed of object $x$ and of pan \#1 as being an extended

Bitbol 1988). 
object and we denote it as $(x, 1)$. The possible observations of an experiment are pictured in Figure 5. You may observe that " $x$ on pan \#1 is lower than $y$ on pan \#2", which is written $(x, 1) \succ_{3}(y, 2)$. You may observe that " $y$ on pan \#2 is lower than $x$ on pan $\# 1$ ", which is written $(y, 2) \succ_{3}(x, 1)$. Finally, you observe that "the balance is at equilibrium when $x$ is on pan \#1 and $y$ on pan \#2", written $(x, 1) \sim_{3}(y, 2)$, if and only if none of the two previous outcomes are observed. Intuitively, the analysis of this setting is identical to the one of the first setting. This is because such an intuitive analysis is carried out from the point of view of the observer and, from such a point of view, calling the arms "left" and "right" or "pan \#1" and "pan \#2" does not make any difference. We will now see that that the formalization is however very different.

In order to reflect observation properly, we cannot simply define the relations $\succ_{3}$ on the set $A \times\{1,2\}$. We want to reflect formally that, for instance, $(x, 1) \succ_{3}$ $(y, 1)$ is neither true or false but simply not observable because $x$ and $y$ are not on two different pans. We therefore need a slightly modified definition of what a relation is.

Let $B=\left(A_{1} \times A_{2}\right) \cup\left(A_{2} \times A_{1}\right)$ where $A_{i}=A \times\{i\}$ with $i \in\{1,2\}$. We define $\succ_{3}$ as a subset of $B$ and we say that $\succ_{3}$ is restrained $d^{9}$ to $B$. We can define the equilibrium relation $\sim_{3}$ from the relation $\succ_{3}$ as: for all $(x, y) \in B$, for all $i \in\{1,2\},(x, i) \sim_{3}(y, 3-i) \Leftrightarrow\left((x, i) \nsucc_{3}(y, 3-i)\right.$ and $\left.(y, 3-i) \nsucc_{3}(x, i)\right)$. As in model 1, the relation $\succsim_{3}$ designates the union of the relations $\succ_{3}$ and $\sim_{3}$ and is defined as, for all $x, y \in A$ and all $i \in\{1,2\}:(x, i) \succsim_{3}(y, 3-i) \Leftrightarrow\left((x, i) \succ_{3}\right.$ $(y, 3-i)$ or $\left.(x, i) \sim_{3}(y, 3-i)\right)$.

As in the other models, the relation $\succ_{3}$ is positive and super-archimedean, and the relations $\succ_{3}$ and $\sim_{3}$ are scale-invariant. However, in this model, the relation $\succ_{3}$ is asymmetric $\left(\sim_{3}\right.$ is symmetric by construction $) .{ }^{10}$ Finally, remark that these relations remain not independent.

As one may have guessed, this model leads to a somehow standard (i.e. not biased) representation:

Theorem 3 Let $A$ be a commutative semigroup. Let $A_{i}=A \times\{i\}(i \in$ $\{1,2\})$ and $B=\left(A_{1} \times A_{2}\right) \cup\left(A_{2} \times A_{1}\right) \subset A \times\{1,2\}$. Let $\succ_{3}$ be a non-trivial binary relation restrained to $B$ that is asymmetric, positive, scale-invariant and super-archimedean. Suppose that $A$ is homogeneous. Then there exist a function $\varphi: A \rightarrow \mathbb{R}_{>0}$ and two numbers $\beta_{1}, \beta_{2} \in \mathbb{R}_{>0}$ such that, for all $x, y \in A$, all $i \in\{1,2\}$, we have

$$
\begin{aligned}
(x, i) \succ_{3}(y, 3-i) & \Longleftrightarrow \beta_{i} \varphi(x)>\beta_{3-i} \varphi(y), \\
\varphi(x \circ y) & =\varphi(x)+\varphi(y),
\end{aligned}
$$

\footnotetext{
${ }^{9}$ For all $x, y \in A$, for all $i \in\{1,2\},(x, i) \succ_{3}(y, 3-i) \Leftrightarrow((x, i),(y, 3-i)) \in B\left(\succ_{3}\right) \subseteq B$. Remark that $B \subset(A \times\{1,2\})^{2}$ hence $\succ_{3}$ is not a relation defined on $A \times\{1,2\}$.

${ }^{10}$ Because the relation is restrained, the formulation of the transitivity property does not make much sense at this stage.
} 


$$
\beta_{1}+\beta_{2}=1
$$

Moreover, the pair $\left(\beta_{1}, \beta_{2}\right)$ is unique and the function $\varphi$ is unique up to multiplication by a positive constant.

Proof. Define the binary relation $\sim_{3}$ restrained on $B$ by: for all $(x, y) \in B$, for all $i \in\{1,2\},(x, i) \sim_{3}(y, 3-i) \Leftrightarrow\left((x, i) \nsucc_{3}(y, 3-i)\right.$ and $\left.(y, 3-i) \nsucc_{3}(x, i)\right)$. Define also the relation $\succsim_{3}$ restrained on $B$ as: for all $(x, y) \in B$, for all $i \in\{1,2\}$ : $(x, i) \succsim_{3}(y, 3-i) \Leftrightarrow\left((x, i) \succ_{3}(y, 3-i)\right.$ or $\left.(x, i) \sim_{3}(y, 3-i)\right)$. Now, from $\succ_{3}$, we define two binary relations $\succ_{3}^{1}$ and $\succ_{3}^{2}$ on $A$ :

$$
x \succ_{3}^{i} y \Leftrightarrow(x, i) \succ_{3}(y, 3-i) .
$$

Both relations verify conditions of Theorem 1 . Hence, for $i=1,2$, there exists a ratio-scale $\varphi_{i}: A \rightarrow \mathbb{R}_{>0}$ and a constant $\alpha_{i} \in \mathbb{R}_{>0}$ such that

$$
x \succ_{3}^{i} y \Leftrightarrow \beta_{i} \varphi_{i}(x)>\left(1-\beta_{i}\right) \varphi_{i}(y) .
$$

Because each $\varphi_{i}$ is unique up to multiplication by a positive scalar, we can suppose that $\varphi_{1}=\varphi_{2}=\varphi$. Moreover, for $i=1,2$, define the binary relation $\succsim_{3}^{i}$ on $A$ by

$$
x \succsim_{3}^{i} y \Leftrightarrow(x, i) \succsim_{3}(y, 3-i) .
$$

We have

$$
x \succsim_{3}^{i} y \Leftrightarrow y \nsucc_{3}^{3-i} x .
$$

Since $x \succ_{3}^{i} y \Rightarrow x \succsim_{3}^{i} y$, we have

$$
\beta_{i} \varphi(x)>\left(1-\beta_{i}\right) \varphi(y) \Rightarrow\left(1-\beta_{3-i}\right) \varphi(x) \geqslant \beta_{3-i} \varphi(y),
$$

which is only possible if $\frac{1-\beta_{1}}{\beta_{1}}=\frac{\beta_{2}}{1-\beta_{2}}$, i.e. if $\beta_{2}=1-\beta_{1}$. The uniqueness conditions are clear.

Naturally, the numbers $\beta_{1}$ and $\beta_{2}$ are interpreted as measuring the distance between the fulcrum and pans $\# 1$ and $\# 2$ respectively. In this model, these numbers are assigned to a variable that was part of the primitives (namely the two pans). We have here an instance of conjoint measurement, which is indeed close to the distributive triples in Luce and Narens (1985). To make the standard nature of the representation even more explicit, we define the function $\Phi: A \times\{1,2\} \rightarrow$ $\mathbb{R}_{>0}$ as

$$
\Phi(x, i)=\beta_{i} \varphi(x) .
$$

Then, the binary relation $\succsim_{3}$ restrained on $B$ can be uniquely extended to a relation $\succsim_{3}$ that is complete and transitive (i.e. a weak order) on $A \times\{1,2\}$. For all $x, y \in A$ and all $i, j \in\{1,2\}$,we let

$$
(x, i) \succsim_{3}(y, j) \Leftrightarrow \Phi(x, i) \geqslant \Phi(y, j) .
$$


In this manner, it is possible to obtain a standard representation without a bias but with a two-attribute function: one attribute for the object and one for its extension, i.e. the pan of the balance on which it is placed. Finally, when the interaction between the objects and the balance can be part of the definition of objects, then the representation of the biased balance is not really biased!

\section{Conclusion}

What would have happened had Apollo not taken his scales by the middle? What do we observe with a biased measuring device? How can we formalize it and how interpretation relate formalism and observation?

This paper provides a rigorous analysis of experiments where objects and a dissymmetric measuring device combine to produce an observable phenomenon. It illustrates how measurement is possible even when the observed relation between objects is incomplete, intransitive or does not verify independence. It also shows how interpretation of observation may lead to a formalism that is dependent on the observer (model 1) or to some indeterminacy in observable behavior (model 2). Finally, it clarifies minimal conditions under which it is possible to treat the interaction with the measuring device as one dimension of some "extended objects" (model 3). The resulting form of "biased measurement" illustrated by the biased balance extends measurement to relational structures that cannot be represented by a function only, but can be represented by a function and a bias. The biased balance also allows to study the relation between measurement and empirical observation in more details. It illustrates how a biased measuring device which is not directly observable influences the observed relation between objects, and it shows how such a measuring device can be measured. In this respect, it provides an intuitive grasp to the distinctive roles played by the observer, the measuring device and the objects, notions which usually pertain to the theory of quantum mechanics (e.g. von Neumann 1955) or to the philosophy of science (e.g. Suppes 1993).

A formal limitation of this paper resides in the homogeneity assumption. We hence work with Bertrand Lemaire on proving similar representation theorems in a non-homogeneous setting. In terms of the biased balance, this would clarify whether such a device can theoretically distinguish between pairs in a non-homogeneous set, such as for instance cubes whose aretes are of length 1 and spheres of diameter 1. Also, I believe that a link with probabilistic models should also be of interest. Finally, Theorem 3 may lead to an interesting generalization, for instance considering the case of a balance with $n$ pans ${ }^{11}$.

\footnotetext{
${ }^{11}$ This would generalize of the notion of a Grassman structure (see Suppes et al. 1989, p. 229)
} 
Finally, I believe that the biased balance can also be a powerful model for the study of biased judgments. Human beings cannot be viewed as deities who judge things without biases, an assumption which is however the cornerstone of rational behavior as maximization of a utility function. Human judgements involve both some observable objects and the subject himself, who is looking at the objects with his specific values, in a manner that can be specific to the situation at hand. It is as if the subject constructs his preferences by positioning himself towards the objects he judges. These models of the biased balance should help to better reflect how such attitudes influence preferences. At last, it could also provide a measurement theoretic approach for studies that combine observation of behavior with observation of the human brain (De Martino et al. 2006).

\section{References}

Bitbol, M. (1988). The concept of measurement and time symmetry in quantum mechanics. Philosophy of Science, 55, 349-375.

Boumans, M. (2005). Measurement in economic systems. Measurement, 38, 275284.

Campbell, N. R. (1957). Foundations of Science. New York: Dover Publications. Carnap, R. (1966/1995). An introduction to the philosophy of science. New York: Dover Publications.

Chevalier, J. and A. Gheerbrant (1969). Dictionnaire des symboles. Paris: Robert Laffont.

De Martino, B., Kumaran, D., Seymour, B, Dolan, R.J. (2006). Frames, Biases, and Rational Decision-Making in the Human Brain. Science, 313: 684-687.

De Miguel, J.R., Candeal, J.C. and E. Indurain (1996). Archimedeaness and additive utility on totally ordered semigroups. Semigroup forum, 52: 335-347.

Ellis, B. (1966). Basic Concepts of Measurement. Cambridge: Cambridge University Press.

Krantz, D.H., Luce, R. D., Suppes, P. and Tversky, A. (1971). Foundations of measurement (Vol. I Additive and Polynomial Representations). San Diego: Academic Press.

Fuchs, L. (1963). Partially ordered algebraic systems. Pergamon Press. 1963.

Heath, T.L. (ed) (1897). The Works of Archimedes with the Method of Archimedes. New York: Dover.

Kahneman, D. and Tversky, A. (2000). Choices, Values and Frames. New York: Cambridge University Press.

Kish, B. (1965). Scales and weights: a historical outline. New Haven and London: Yale University Press.

Lemaire, B. and Le Menestrel, M. (2006). Homothetic interval orders. Discrete Mathematics, 306, 1669-1683.

Le Menestrel, M. and Lemaire, B. (2004). Biased extensive measurement: the homogeneous case. Journal of Mathematical Psychology, 48, 9-14. 
Le Menestrel, M. and Lemaire, B. (2006a). Biased extensive measurement: the general case. Journal of Mathematical Psychology, 50, 570-581.

Le Menestrel, M. \& Lemaire, B. (2006b). Ratio-scale measurement with intransitivity or incompleteness: the homogeneous case. Theory and Decision, 60, 207-217.

Le Menestrel, M. \& Van Wassenhove, L. (2001). The Domain and Interpretation of Utility Functions: An Exploration. Theory and Decision 51, 329-349.

Luce, R. D. and Narens, L. (1985). Classification of Concatenation Structures according to Scale Type. Journal of Mathematical Psychology, 29, 9-72.

Mari, L. (2000). Beyond the representational viewpoint: a new formalization of measurement. Measurement, 27, 71-84.

Mari, L. (2005). The problem of foundations of measurement. Measurement, 38, 259-266.

Michell, J. (2005). The logic of measurement: a realist overview. Measurement, 38, 285-294.

Michell, J. and C. Ernst (1996). The axioms of quantity and the theory of measurement. Translated from Part I of Otto Hölder's German Text "Die Axiome der Quantität and die Lehre vom Mass". Journal of Mathematical Psychology 40, 235-252.

Moody, E. A. and M. Clagett (1960). The Medieval science of weights (scientia de Ponderibus). Madison: The university of Wisconsin Press.

Narens, L. (1985). Abstract Measurement Theory. Cambridge: MIT Press.

Quine, W.V. (1975). On Empirically Equivalent Systems of the World. Erkenntnis, 9, 313-328.

Roberts, F. S. (1979). Measurement Theory with applications to decision making, utility and the social sciences. Reading: Addison-Wesley.

Schwager, K.W. (1991). The representational theory of measurement: an assessment. Psychological Bulletin, 110, 618-626.

Sen, A. (1986), Information and invariance in normative choice. In Social Choice and Public Decision Making (pp. 29-55). Cambridge, UK: Cambridge University Press.

Slovic, P. (1995). The construction of preferences. American Psychologist, 50, 364-271.

Stevens, S. (1946). On the theory of scales of measurement. Science, 103, 122136.

Suppes, P. (1980). Limitations of the axiomatic method in ancient Greek mathematical sciences. In Pisa Conference Proceedings (J. Hintikka, D. Gruender, and E. Agazzi (eds), D. Reidel Publishing Company, Vol. I, 197-213.

Suppes, P. (1993). Models and Methods in the Philosophy of Science: Selected Essays. Dordrecht: Kluwer Academic Publishers.

Suppes, P., Krantz, D.H., Luce, R. D. and Tversky, A. (1989). Foundations of measurement (Vol. II Geometrical, Threshold, and Probabilistic Representations). San Diego: Academic Press. 
von Neumann, J. (1955). Mathematical foundations of quantum mechanics. Princeton University Press. 